\title{
BMJ Open Inequalities in access to cardiac rehabilitation after an acute coronary syndrome: the EPiHeart cohort
}

\author{
Marta Viana, ${ }^{1,2}$ Andreia Borges, ${ }^{1}$ Carla Araújo, ${ }^{1,3}$ Afonso Rocha, ${ }^{4}$ Ana I Ribeiro, ${ }^{1}$ \\ Olga Laszczyńska, ${ }^{1}$ Paula Dias, ${ }^{5}$ Maria J Maciel, ${ }^{5}$ Ilídio Moreira, ${ }^{3}$ Nuno Lunet, ${ }^{1,6}$ \\ Ana Azevedo ${ }^{1,6,2}$
}

\begin{abstract}
To cite: Viana M, Borges A, Araújo $\mathrm{C}$, et al. Inequalities in access to cardiac rehabilitation after an acute coronary syndrome: the EPiHeart cohort. BMJ Open 2018;8:e018934. doi:10.1136/ bmjopen-2017-018934

- Prepublication history for this paper is available online. To view these files, please visit the journal online (http://dx.doi. org/10.1136/bmjopen-2017018934).
\end{abstract}

$M V$ and $A B$ contributed equally.

Received 1 August 2017

Revised 26 0ctober 2017

Accepted 27 October 2017

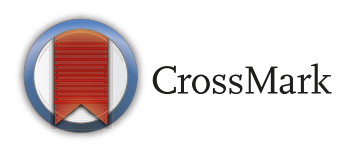

${ }^{1}$ EPIUnit, Instituto de Saúde Pública, Universidade do Porto, Porto, Portugal

${ }^{2}$ Centro de Epidemiologia Hospitalar, Centro Hospitalar de São João, Porto, Portugal ${ }^{3}$ Serviço de Cardiologia, Centro Hospitalar de Trás-os-Montes e Alto Douro, Vila Real, Portugal ${ }^{4}$ Serviço de Medicina Física e Reabilitação, Centro Hospitalar de São João, Porto, Portugal

${ }^{5}$ Serviço de Cardiologia, Centro Hospitalar de São João, Porto, Portugal

${ }^{6}$ Departamento de Ciências da Saúde Pública e Forenses e Educação Médica, Faculdade de Medicina da Universidade do Porto, Porto, Portugal

Correspondence to

Marta Viana;

marta.viana@ispup.up.pt

\section{ABSTRACT}

Objectives To estimate cardiac rehabilitation (CR) referral and participation rates among patients with acute coronary syndrome (ACS) and to identify their determinants, in two Portuguese regions.

Design Prospective cohort study.

Setting Patients consecutively admitted to the cardiology department of two hospitals, one in the district of Porto and one in the north-east region (NER) of Portugal, were enrolled in the EPIHeart cohort and then followed up for 6 months.

Participants Between August 2013 and December 2014, 939 patients were included in the cohort, and 853 were re-evaluated at 6-month follow-up.

Outcome measures Referral rate was defined as the proportion of eligible patients who were referred to a CR programme, whereas participation rate was defined as the proportion of eligible patients who completed a CR programme, as was recommended by their physicians. Results Patients referred were $32.3 \%$ and $10.7 \%$ of those eligible in Porto and NER, respectively. In both regions, referral to $\mathrm{CR}$ decreased with age and with longer travel times to CR centres and increased with education or social class. At follow-up, 128 patients from Porto $(26.2 \%$ of those eligible and $81.0 \%$ of those referred) and 26 from NER $(7.1 \%$ of those eligible and $66.7 \%$ of those referred) reported actually participating in a CR programme. In Porto, the main barriers to participation were the long time until a programme was available and lack of perceived benefit. Patients in NER identified distance to CR and costs as the main barriers.

Conclusions CR remains clearly underused in Portugal, with major inequalities in access between regions. Achieving equitable and greater use of $\mathrm{CR}$ requires a multilevel approach addressing barriers related to healthcare system, providers and patients in order to improve provision, referral and participation.

\section{INTRODUCTION}

Cardiac rehabilitation (CR) programmes are currently recognised as an integral part of the approach for secondary prevention of coronary heart disease (CHD) and considered a class I recommendation in current clinical guidelines by the American Heart Association,

\section{Strengths and limitations of this study}

- This study assessed the frequency and determinants of referral and participation to cardiac rehabilitation (CR), among urban and rural settings.

- Identifying target groups and geographic areas where access is more limited is essential to establish clear strategies to reduce inequalities in the use of healthcare services.

- Our results may not be generalised to other settings with different characteristics, although the study was conducted in two hospitals attending patients with contrasting use of $\mathrm{CR}$.

the American College of Cardiology and the European Society of Cardiology for treatment of patients with CHD. ${ }^{1-4}$

A recent Cochrane systematic review showed that CR reduces cardiovascular mortality and hospitalisations while improving quality of life. ${ }^{5}$ This evidence was subsequently extended by a multicentre cohort of patients with acute myocardial infarction (AMI) showing that participation in CR was associated with $41 \%$ lower hazard of all-cause mortality. ${ }^{6}$ Moreover, CR has been described as a cost-effective intervention, ${ }^{78}$ with cost-effectiveness ratios similar to the use of angiotensin converting enzyme (ACE) inhibitors and statins. ${ }^{8}$

Despite the robust evidence supporting the use of CR, it clearly remains underused worldwide. $^{9}$ In Portugal, the proportion of eligible patients enrolled in rehabilitation programmes has increased from 3\% in 2007 to $8 \%$ in $2013,{ }^{10}{ }^{11}$ though still far from the $30 \%$ target set by the National Health Plan for 2010. ${ }^{12}$ The implementation of CR in Portugal is constrained by insufficient supply of CR services and large geographical disparities in their distribution, with CR centres concentrated mainly in large urban areas of the country. ${ }^{1011}$ The aim of the present study was to estimate the proportion of patients 
with acute coronary syndrome (ACS) who were referred and who actually participated in a CR programme, comparing two regions in the north of Portugal. The work also intended to identify determinants of referral and to describe barriers to participation.

\section{METHODS}

\section{Setting}

The present study evaluated patients with an ACS enrolled in the EPIHeart cohort, who were admitted to two hospitals (São João Hospital and São Pedro Hospital) in the north of mainland Portugal. São João Hospital is a university hospital located in the Porto municipality, which serves as first-line service part of the population of the Porto district (mainly coastal and urban), whereas São Pedro Hospital is a tertiary-level hospital located in the Vila Real municipality serving, as first-line or referral target, the population of the north-east region (NER) of Portugal (mainly rural and located in the inner land), which covers the districts of Vila Real, Bragança and some municipalities of the Viseu district. Since the EPIHeart cohort was set up to assess inequalities in CHD management and outcomes in urban and rural settings in Portugal, the selection of these regions intended to capture the variation in CHD management and outcomes across populations with different sociodemographic and clinical characteristics.

In the four districts considered in this study, there are eight CR centres available, seven in the Porto district and one in the NER. ${ }^{11}$ In the Porto district, there are four public centres, located within the facilities of public hospitals, and three private centres, located in privately owned healthcare organisations. In the Porto district, the first CR programme was implemented in 1992 in a private clinic, and the last one was implemented in 2008 in São João Hospital. In the NER, there is only one private centre available, which was launched in 2012.

The location of the study area is depicted in figure 1A.

\section{Study population}

Between August 2013 and December 2014, patients consecutively admitted to the cardiology departments of the two hospitals involved in the study were enrolled in the EPIHeart cohort and then followed up for 6 months. Hospitalised patients were invited to participate in the study by trained staff nurses or physicians in charge, who gave written information, explained the study verbally and obtained written informed consent. The inclusion criteria were to be admitted with a diagnosis of ACS type I, aged $\geq 18$ years, expected to have a length of stay longer than 48 hours and living inside the hospitals' catchments area. Exclusion criteria were non-confirmed diagnosis of ACS, death, discharge or transfer prior to interview invitation and inability to answer the questionnaires, due to clinical instability, no domain of Portuguese language, hearing problems or cognitive impairment, as evaluated by the interviewer. Of the 1297 patients initially considered, 286 were excluded, and 72 refused to participate (7.1\% of those invited, $4 \%$ in the Porto district and $11 \%$
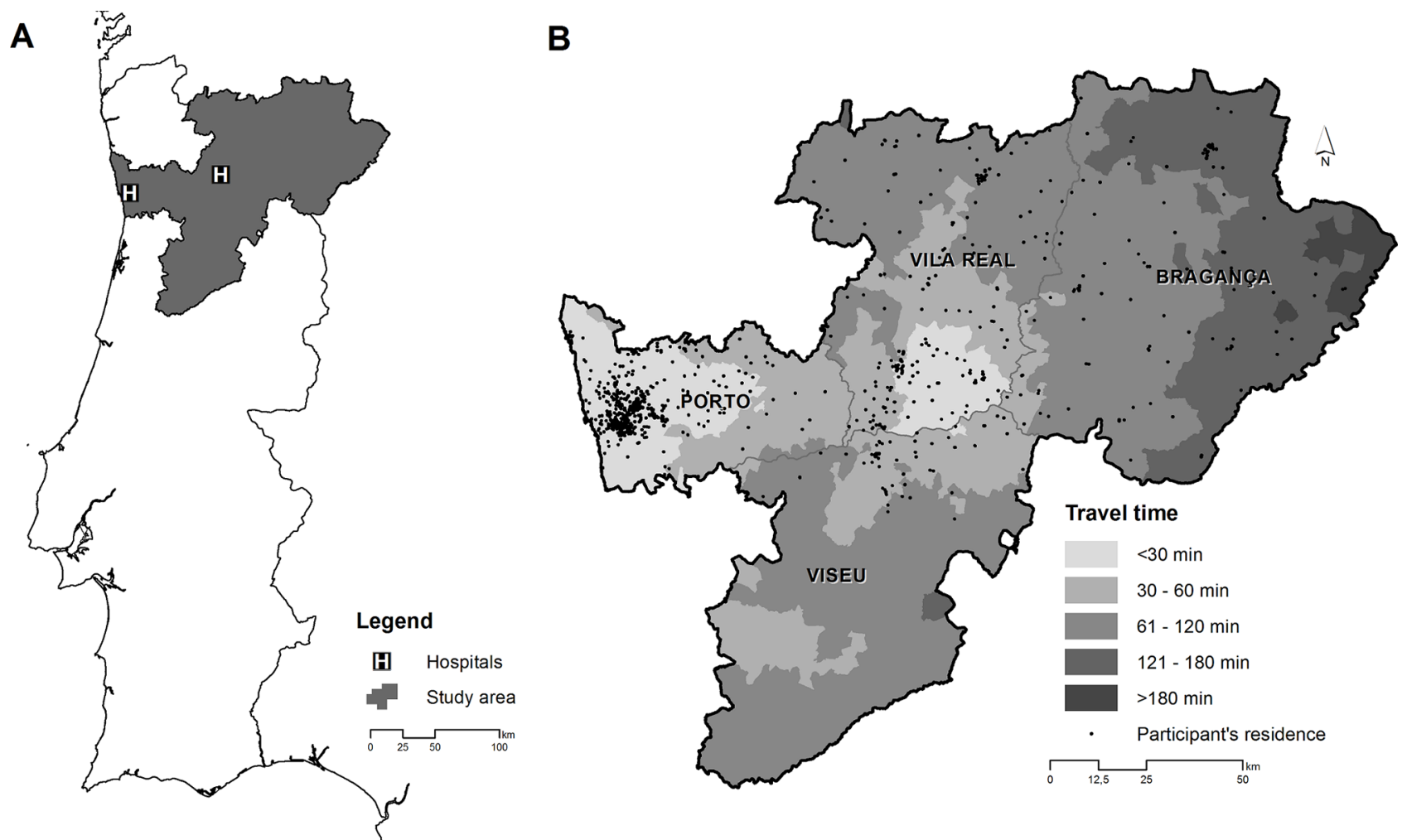

Figure 1 EPIHeart cohort study setting. (A) Study area and location of the hospitals. (B) Travel times from patients' home to the nearest cardiac rehabilitation centre. 
in the NER), thus the final sample included 939 patients. Patients who refused to participate in the study were significantly older $(72.7$ years vs 64.2 years, $\mathrm{P}<0.001)$ and had lower education than those who participated (4 years of schooling or less: $59.4 \%$ vs $77.6 \%, \mathrm{P}=0.003$ ), whereas there was no significant difference in the ACS types (ST-segment elevation myocardial infarction (STEMI): $37.1 \%$ vs $37.4 \%, \mathrm{P}=0.961$ ). At the 6-month follow-up, 51 patients were dead, 18 patients were not evaluated due to invalid contact details and 17 refused to continue in the study, hence 853 patients were included in the current analysis. Non-participants in the follow-up were older (non-participants due to death: 75.3 years vs non-participants due to refusals and losses: 64.1 years vs participants: 63.5 years, $\mathrm{P}<0.001)$ and had lower education $(\leq 4$ years of schooling) than those who participated (non-participants due to death: $82.6 \%$ vs participants due to refusals and losses: $71.9 \%$ vs participants: $57.7 \%, \mathrm{P}<0.001)$.

The study protocol complied with the Declaration of Helsinki and was approved by the Ethics Committee of both hospitals involved.

\section{Data collection}

At baseline, a face-to-face interview was conducted by trained researchers to collect data about sociodemographic characteristics, and the patients' medical records were reviewed to collect clinical data.

Sociodemographic data included age, sex, home address, marital status, health insurance coverage, subjective social class, schooling years, main activity, occupation and monthly household income. The marital status was considered partnered for married patients or living in civil union and unpartnered for patients who were single, separated, divorced or widow(er). Health insurance coverage comprised health subsystems and private health insurance. Subjective social class was assessed by asking patients to identify their social class category from a list provided, that included lower class, lower-middle class, upper-middle class and upper class. Occupations were classified into major professional groups, according to the Portuguese National Classification of Occupations 2010 integrated in the International Standard Classification of Occupations $2008^{1314}$ and grouped into three categories: upper white collar (executive civil servants, industrial directors and executives, professionals and scientists and middle management and technicians), lower white collar (administrative and related workers and service and sales workers) and blue collar (farmers and skilled agricultural, fisheries workers, skilled workers, craftsmen and similar, machine operators and assembly workers and unskilled workers). Armed forces were not considered for this classification. Retired, disabled and housewives were classified considering their previous main occupation, when applicable.

Clinical data included risk factors and comorbidities, clinical characteristics at admission, medical procedures, left ventricular systolic dysfunction (LVSD) and type of ACS. LVSD was considered present when there was a qualitative description consistent with moderate or severe systolic dysfunction in the last completed echocardiography during the hospital stay. The type of ACS was used as clinically defined in discharge notes and classified as STEMI and non-ST-elevation ACS (NSTEACS), with the latter including unstable angina, non-ST-elevation AMI, subacute MI and non-classified ACS.

Six months after the index event, patients were contacted by telephone and asked whether they were referred to CR following the event and whether they attended the CR programme as was recommended by their physician. Patients who attended and completed the CR programme were asked to identify the CR centre and the number of sessions attended. Patients who did not attend or complete the programme were asked about the reasons for non-participation, through the selection of one or more of the following options: (1) cost, (2) distance from home to the CR centre, (3) limited time availability, (4) lack of perceived benefit, (5) delay in enrolment in a CR programme due to lack of vacant places and (6) other reason. Referral rate was defined as the proportion of eligible patients who were referred to a CR programme, whereas participation rate was defined as the proportion of eligible patients who completed a CR programme, as was recommended by their physicians.

In order to compute travel time to the nearest CR centre, descriptive data about the available CR centres in Portugal were collected, based on the results of the national surveys on CR centres. ${ }^{11}$ All patients and CR centres were georeferenced according to the address using ArcGIS Online World Geocoding Service and Google Maps. The shortest road distance (in min) from the patients' home to the nearest CR centre was calculated with ArcGIS V.10.4.1 and the Network Analyst extension, using an updated street network dataset provided by the Environmental Systems Research Institute.

\section{Statistical analysis}

Continuous variables are described as mean and SD, whereas categorical variables are presented as absolute frequencies and percentages. Categorical and continuous variables were compared using the $\chi^{2}$ test and the two-tailed Student's t-test, as appropriate. The relation between patients' characteristics and referral to CR was quantified through the calculation of ORs and 95\%CIs, using unconditional logistic regression. Analyses were conducted for all patients and also stratified according to referral hospital. Starting from models with all candidate predictors ( $\mathrm{P}$ value $<0.15$ ), based on results from bivariate analysis, independent predictors were identified after stepwise backward elimination $(\mathrm{P}$ value $>0.05)$. All tests were two tailed, and a $\mathrm{P}$ value $<0.05$ was considered statistically significant. Data were analysed using STATA V.11 for Windows (StataCorp).

\section{RESULTS}

Among the 853 patients included in this study (mean age $63.5 \pm 12.9$ years, $75.2 \%$ men), $489(57.3 \%)$ were 
from the Porto district, and $364(42.7 \%)$ were from the NER of Portugal. Baseline characteristics are summarised in table 1. Compared with patients from the NER, those from Porto were more often male (79.1\% vs $69.8 \%, \mathrm{P}=0.002)$, younger (61.6 years vs 66.7 years, $\mathrm{P}<0.001)$, more frequently employed $(34.3 \%$ vs $22.5 \%, \mathrm{P}<0.001)$ and had higher levels of education ( $>4$ schooling years: $50.5 \%$ vs $31.4 \%, \mathrm{P}<0.001$ ), social class (higher-middle/high: $9.0 \%$ vs $4.4 \%, \mathrm{P}=0.001$ ) and household income ( $€ € 1500: 19.0 \%$ vs $11.8 \%, \mathrm{P}=0.014$ ). Hypertension ( $72.5 \%$ vs $63.0 \%, \mathrm{P}=0.003)$, dyslipidaemia ( $66.8 \%$ vs $58.7 \%, \mathrm{P}=0.016)$, chronic heart failure $(9.1 \%$ vs $4.7 \%, \mathrm{P}=0.012)$ and impaired renal function at admission $(9.9 \%$ vs $5.7 \%, \mathrm{P}=0.022)$ were more frequent among patients who lived in the NER, whereas current smoking $(38.5 \%$ vs $19.5 \%, \mathrm{P}<0.001)$ and peripheral arterial disease $(6.3 \%$ vs $3.3 \%, \mathrm{P}=0.045)$ were more frequent among those who lived in Porto. The proportion of patients discharged with a diagnosis of STEMI was lower in the NER than in the Porto district $(33.2 \%$ vs $42.7 \%, \mathrm{P}=0.005$ ). Patients who lived in the NER were more likely to need a travel time to CR greater than 30 min compared with those who lived in the Porto district $(82.1 \%$ vs $5.3 \%, \mathrm{P}<0.001)$. Travel times to the nearest CR centre are illustrated in figure 1B.

From the whole study sample, 158 patients from Porto district were referred by their physicians to attend a CR programme, whereas only 39 from the NER were referred $(32.3 \%$ vs $10.7 \%, \mathrm{P}<0.001)$ (figure 2$)$. In both regions, patients who were male, younger, employed, with a white collar occupation, in higher social class, education and income and with health insurance coverage were more likely to be referred to a CR programme. Those who lived nearest to a CR centre, who were smokers and who were diagnosed with STEMI were also more likely to be referred to rehabilitation, whereas patients with comorbidities, anaemia at admission or impaired renal function were less likely to be referred to CR in both regions (table 2).

Predictors independently associated with CR referral are presented in table 3. In both regions, the probability of referral to CR decreased with age (Porto district: OR 0.95, 95\% CI 0.93 to 0.97 per year of age; NER: OR $0.95,95 \%$ CI 0.91 to 0.98 per year of age) and with longer travel times to CR centres (Porto district: OR 0.18, 95\% CI 0.04 to 0.92; NER: OR $0.18,95 \%$ CI 0.07 to 0.42 ). Patients living in the NER were less likely to be referred to CR, but the differences were not statistically significant, whereas longer travel times were negatively associated with referral. Among patients who lived in the Porto district, current smokers (OR 1.89, 95\% CI 1.12 to 3.20 ), in higher social class (lower-middle: OR 2.20, 95\% CI 1.17 to 4.14; higher-middle: OR 2.30, $95 \%$ CI 0.94 to 5.61), with health insurance coverage (OR 2.11, 95\% CI 1.23 to 3.62) and those who underwent a percutaneous coronary intervention (PCI) (OR 3.24, $95 \%$ CI 1.85 to 5.66 ) were more likely to be referred to $\mathrm{CR}$, whereas those with comorbidities were less likely to be referred ( 1 comorbidity: OR $0.40,95 \%$ CI 0.19 to 0.80 ; $\geq 2$ comorbidities: OR $0.22,95 \%$ CI 0.05 to 1.01$)$. In patients who lived in the NER, higher education (OR 3.10, 95\% CI 1.09 to 8.84) and higher household income (OR 4.83, $95 \%$ CI 1.84 to 12.71 ) were independently associated with referral to CR.

Six months after hospital discharge, 128 patients from Porto and 26 from the NER reported participating in a CR programme (26.2\% vs $7.1 \%$ of those eligible, $\mathrm{P}<0.001 \%$ and $81.0 \%$ vs $66.7 \%$ of those referred, $\mathrm{P}<0.052$ ) (figure 2 ). Among the 43 patients who did not enrol or complete the CR programme, the three major reasons reported for non-participation were the long time until a CR programme was available $(33.3 \%)$, limited time available to participate $(23.3 \%)$ and lack of perceived benefit (20.0\%), according to patients from the Porto district, whereas most patients from the NER reported first the distance from home to the CR centre $(84.6 \%)$, then the cost of CR $(23.1 \%)$ and finally the limited time availability $(7.7 \%)$.

\section{DISCUSSION}

Despite the proven benefits and the international recommendations for CR after an ACS, our results showed that CR remains underused in Portugal, with major inequalities in access between regions. Following an ACS, one-third of the patients from the Porto district were referred to CR, whereas only 1 out of 10 patients from the NER were referred. Yet, of those who were referred, four-fifths from Porto and two-thirds from NER actually completed the CR programme, indicating that low participation was largely explained by low referral. Older patients with comorbidities, with lower socioeconomic status and those living farther from a CR centre were less likely to be referred to $\mathrm{CR}$, whereas patients with health insurance coverage, smokers and those who underwent a PCI were more likely to be referred.

The number of patients enrolled in CR programmes in Portugal in recent years is increasing, ${ }^{10}{ }^{11}$ however, such programmes remain considerably underused, indicating that a large proportion of patients with CHD are missing the opportunity to benefit from CR. Several studies have shown that suboptimal referral patterns represent one of the major causes of poor participation in $\mathrm{CR},{ }^{15}$ which is corroborated by our findings. Although in the Porto district the proportion of patients referred met the target set by the Portuguese Minister of Health, in the NER referral was far from reaching $30 \% .^{12}$ Studies conducted in other countries showed wide international variation, ${ }^{16}{ }^{17}$ however, with much higher overall referral than in Portugal. A meta-analysis that included 241613 patients with CHD reported overall referrals of $45 \%,{ }^{17}$ similarly to those observed in the European Action on Secondary and Primary Prevention through Intervention to Reduce Events (EUROASPIRE) survey, which included 13935 patients with CHD, from 22 European countries. ${ }^{16}$ Moreover, in the USA, data from the initiative Get with Guidelines, including 72817 patients hospitalised after an MI, PCI or cardiac artery bypass graft, showed that $56 \%$ 
Table 1 Baseline characteristics of the study population by region

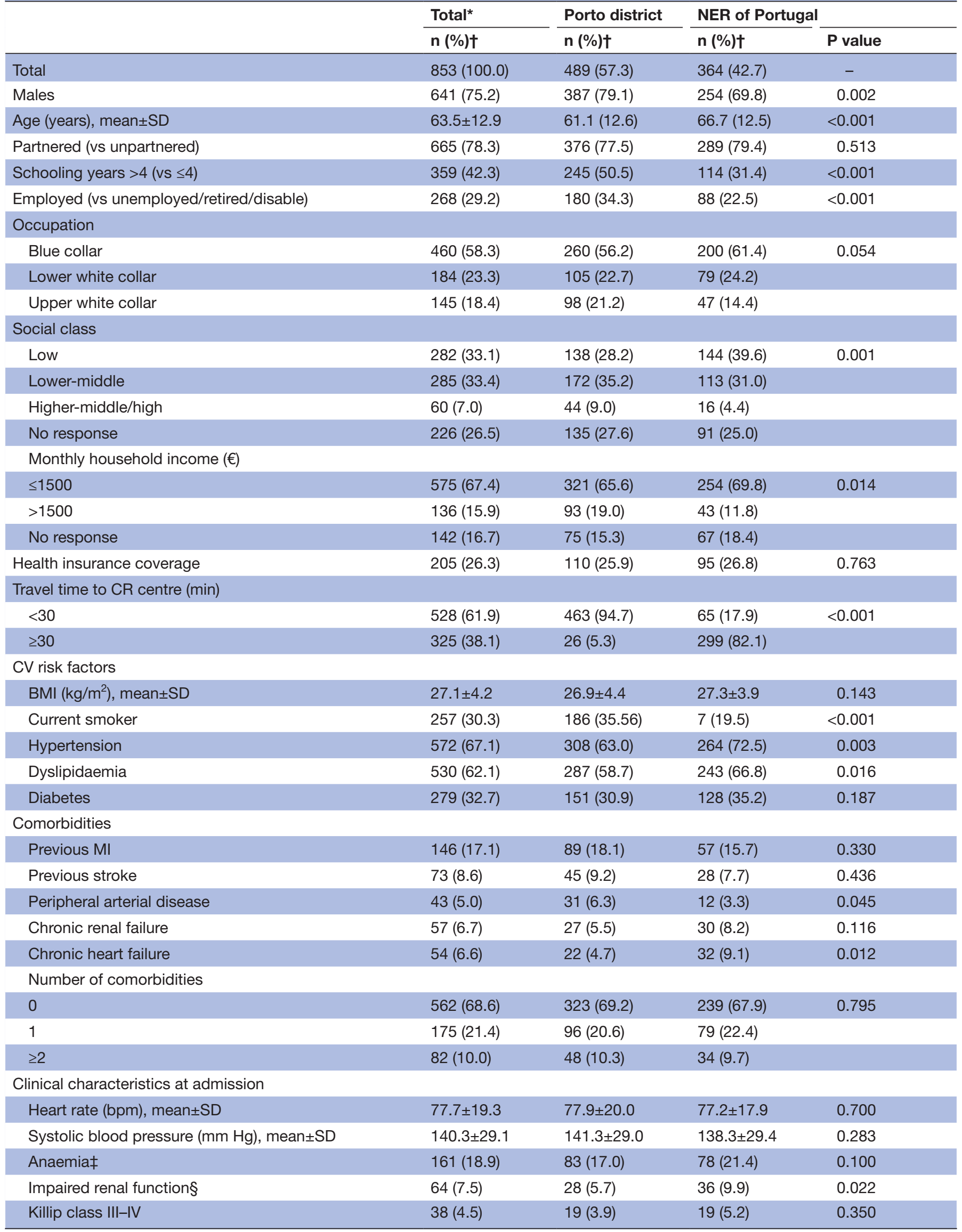


Table 1 Continued

\begin{tabular}{|c|c|c|c|c|}
\hline & \multirow{2}{*}{$\begin{array}{l}\text { Total }^{*} \\
\text { n (\%)† }\end{array}$} & \multirow{2}{*}{$\begin{array}{l}\text { Porto district } \\
\text { n (\%)† }\end{array}$} & \multicolumn{2}{|c|}{ NER of Portugal } \\
\hline & & & n (\%)† & $P$ value \\
\hline Moderate-severe LVSD & $249(30.0)$ & $146(29.9)$ & $103(30.0)$ & 0.973 \\
\hline \multicolumn{5}{|l|}{ Medical procedures } \\
\hline $\mathrm{PCl}$ & $544(63.8)$ & $310(63.4)$ & $234(64.3)$ & 0.789 \\
\hline CABG & $135(15.8)$ & $85(17.4)$ & $50(13.7)$ & 0.149 \\
\hline
\end{tabular}

*Total may not add to 853 due to missing data.

†Results are presented as $\mathrm{n}(\%)$, except if otherwise specified.

$\ddagger$ Anaemia was defined as haemoglobin level $\leq 12 \mathrm{~g} / \mathrm{dL}$ for women and $\leq 13 \mathrm{~g} / \mathrm{dL}$ for men.

§Impaired renal function was defined as serum creatinine level $\geq 1.5 \mathrm{mg} / \mathrm{dL}$.

BMI, body mass index; CABG, cardiac artery bypass graft; CR, cardiac rehabilitation; CV, cardiovascular; LVSD, left ventricular systolic dysfunction; MI, myocardial infarction; NER, north-east region; NSTEACS, non-ST-elevation acute coronary syndrome; PCI, percutaneous coronary intervention; STEMI, ST-segment elevation myocardial infarction.

of those eligible were referred, ${ }^{18}$ whereas recent results of a national survey in England showed $81.5 \%$ of patients referred to CR between 2012 and $2015 .^{19}$

Previous research has demonstrated that there are wide inequalities in CR referral against women, elders and deprived socioeconomic populations. ${ }^{16-18} 2021$ In fact, we found that older patients were less likely to be referred to $\mathrm{CR}$, but no association was found regarding sex. In addition to higher income and education being associated with referral in the NER, our results also showed that patients in the lower social class and with multiple comorbidities were less likely to be referred in the Porto district, reiterating the treatment-risk paradox, ${ }^{22}{ }^{23}$ such that patients at high-risk who may benefit the most from CR are less likely to access it. These findings are particularly worrisome in the current context of population ageing, with an increasing number of comorbidities. Although evidence shows that all patients with CHD benefit from participation in CR, regardless of age or medical comorbidity burden, ${ }^{24}$ physicians may perceive these disadvantaged groups as less likely to take advantage of CR. Several studies that analysed physician factors associated with CR

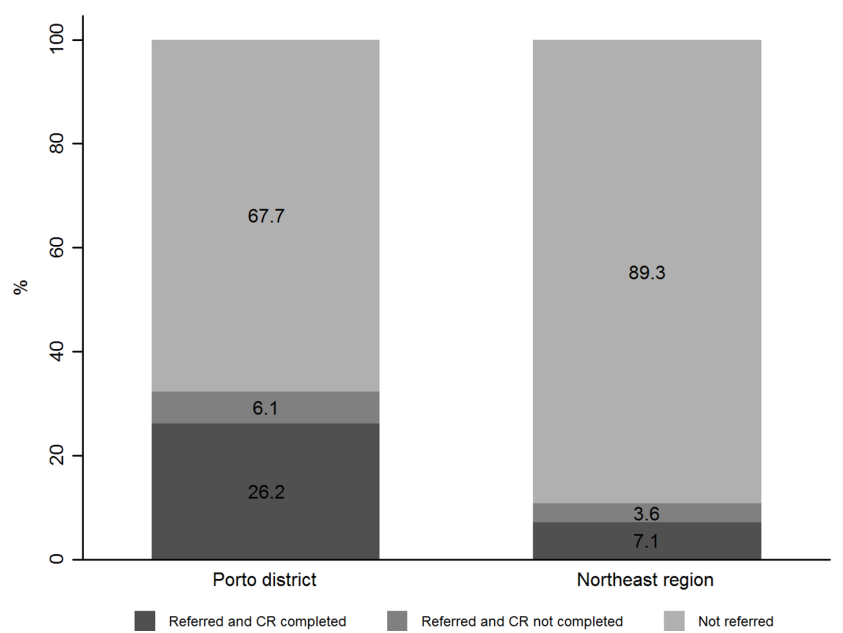

Figure 2 Referral and participation rates to $\mathrm{CR}$ according to region. $\mathrm{CR}$, cardiac rehabilitation. referral pointed out the perceived benefit of CR as one of the most relevant factors affecting referral. ${ }^{25} 26$ Therefore, increasing physicians' awareness about the benefits of CR in higher risk populations is warranted. Besides the importance of physicians' endorsement, implementing systematic referral approaches in which eligible patients are automatically identified and referred to CR have been shown to have a significant impact on increasing referral and enrolment. ${ }^{27}$ Grace et al demonstrated that using a combined automatic referral with patient discussion can achieve referral of over $85 \%$ and enrolment around $74 \% .{ }^{28}$ Moreover, these strategies have been described as having the potential to mitigate disparities in access to CR, namely among patients with lower socioeconomic status. ${ }^{29}$

Our results also showed that patients who live farther from a CR centre were less likely to be referred, in accordance with previous studies that described geographical accessibility, measured by distance, transportation, travel time or costs, as an important barrier to CR referral and participation..$^{25} 30$ The $30 \mathrm{~min}$ travel time threshold has often been used to define accessible healthcare services; ${ }^{31} 32$ however, a study conducted by Brual et al that investigated the travel time threshold affecting specifically CR use found that patients who have a travel time to CR greater than 60 min were less likely to be referred and also to be enrolled in a programme. ${ }^{30}$ In our study, almost half of the patients from the NER lived beyond $60 \mathrm{~min}$ of the CR centre compared with a residual proportion in the Porto district, indicating that patients from the NER face higher geographical barriers to access CR services than patients living in the Porto district. To overcome geographical barriers in access to CR, alternative delivery models, such as home-based programmes, should be considered, particularly for those patients in rural areas. A recent systematic review of data from 17 randomised trials, ${ }^{33}$ which included 2172 patients from 10 countries, compared the effectiveness of home-based to supervised centre-based CR and found similar benefits in terms of mortality, morbidity, quality of life and modifiable risk factors. 
Table 2 Referral to CR after an ACS according to the patients' characteristics by region

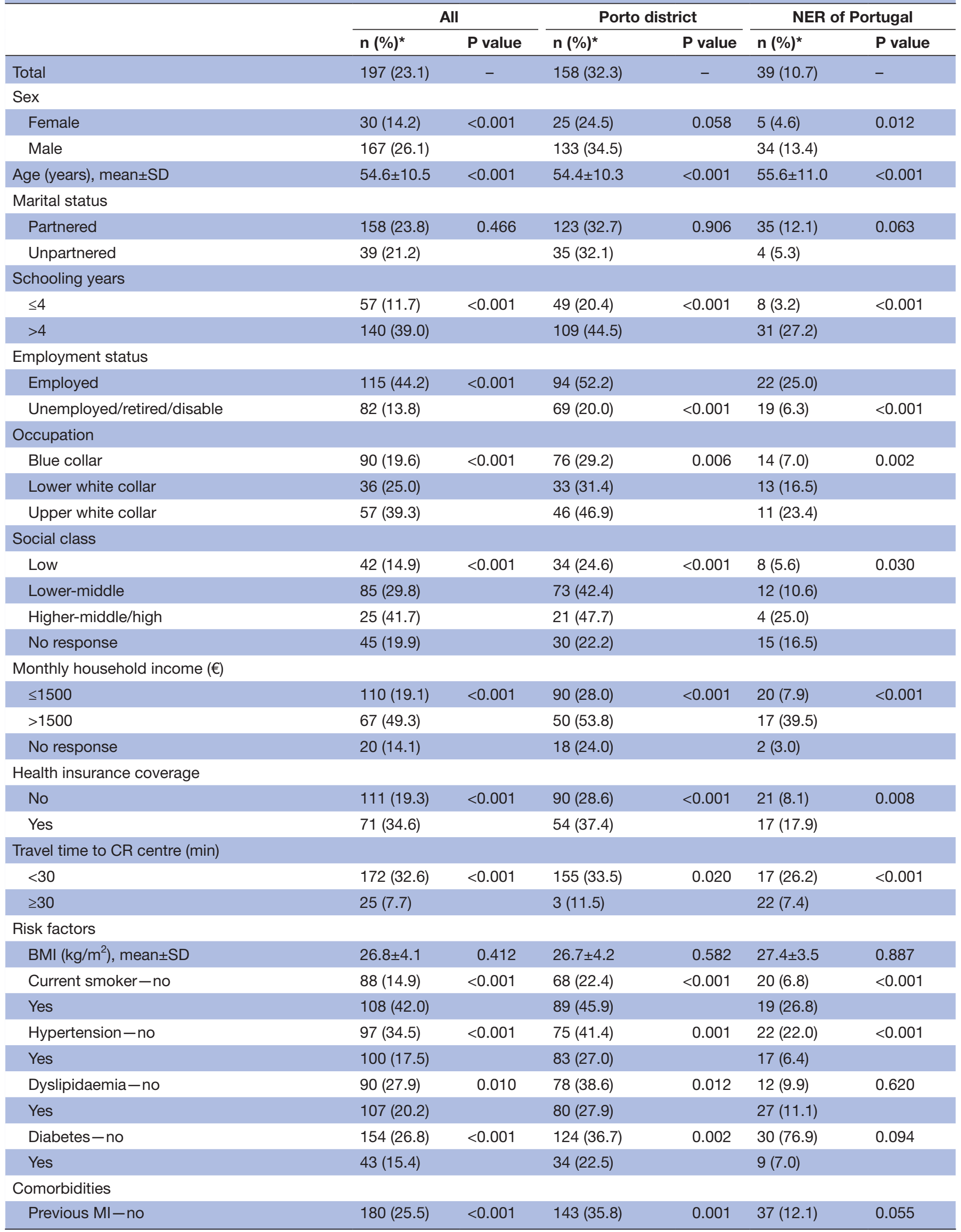


Table 2 Continued

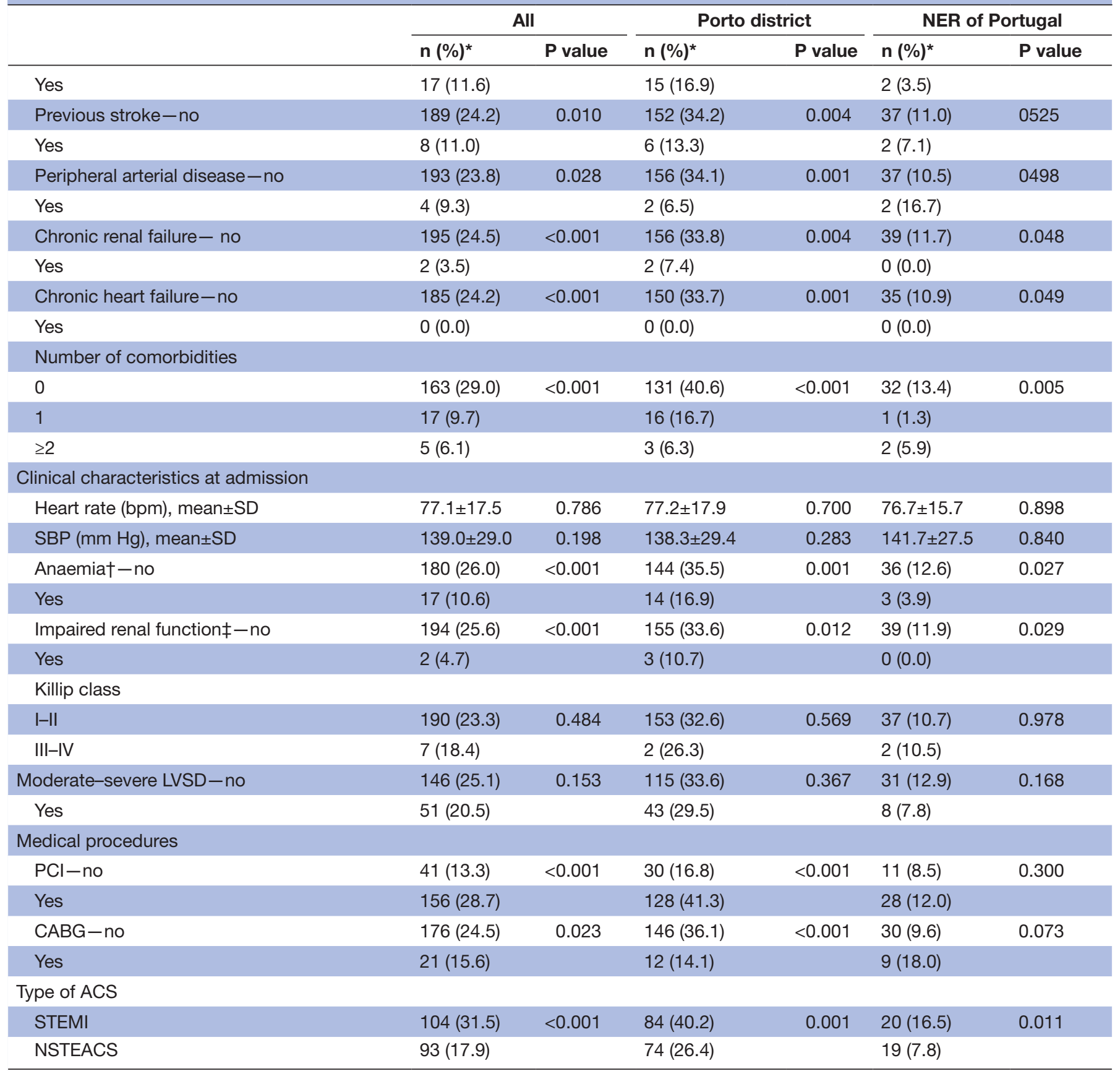

${ }^{*}$ Results are presented as $\mathrm{n}(\%)$, except if otherwise specified.

$\dagger$ Anaemia was defined as haemoglobin level $\leq 12 \mathrm{~g} / \mathrm{dL}$ for women and $\leq 13 \mathrm{~g} / \mathrm{dL}$ for men.

†Impaired renal function was defined as serum creatinine level $\geq 1.5 \mathrm{mg} / \mathrm{dL}$.

ACS, acute coronary syndrome; BMI, body mass index; CABG, cardiac artery bypass graft; CR, cardiac rehabilitation; LVSD, left ventricular systolic dysfunction; MI, myocardial infarction; NER, north-east region; NSTEACS, non-ST-elevation acute coronary syndrome; PCI, percutaneous coronary intervention; SBP, systolic blood pressure; STEMI, ST-elevation myocardial infarction.

Consistent with the results reported in the EUROASPIRE III, ${ }^{16}$ our study showed that a high proportion of referred patients from the Porto district completed the CR programme. Nonetheless, in the NER, the proportion of those failing to complete the programme (33.3\%) contributed to the underuse of CR. According to patients in the NER region, the main barriers to their participation in CR were distance to the centre and financial costs, in line with previous research that identified those as important predictors of non-participation. ${ }^{34}{ }^{35}$ The geographic maldistribution of CR centres is a well-known barrier to access CR in Portugal, with significantly fewer centres in inland areas compared with the coast and in the south compared with the north. ${ }^{10}{ }^{11}$ In the Porto district, there were seven centres, either public hospital-based or private community-based, whereas in the NER, there was 
Table 3 Predictors of CR referral among patients following an acute coronary syndrome by region

Adjusted OR (95\% Cl)

\begin{tabular}{|c|c|c|c|}
\hline & All & Porto district & NER of Portugal \\
\hline \multicolumn{4}{|l|}{ Region* } \\
\hline Porto district & 1 & - & - \\
\hline \multicolumn{4}{|l|}{$\operatorname{Sex}^{*}$} \\
\hline Female & 1 & 1 & 1 \\
\hline \multicolumn{4}{|l|}{ Schooling years (years) } \\
\hline$\leq 4$ & 1 & - & 1 \\
\hline$>4$ & $1.72(1.07$ to 2.76$)$ & - & 3.10 (1.09 to 8.84$)$ \\
\hline \multicolumn{4}{|c|}{ Monthly household income $(€)$} \\
\hline \multicolumn{4}{|l|}{ Social class } \\
\hline Low & - & 1 & - \\
\hline Lower-middle & - & $2.20(1.17$ to 4.14$)$ & - \\
\hline Higher-middle/high & - & $2.30(0.94$ to 5.61$)$ & - \\
\hline No response & - & 1.02 (0.50 to 2.08$)$ & - \\
\hline \multicolumn{4}{|c|}{ Health insurance coverage } \\
\hline No & - & 1 & - \\
\hline Yes & - & 2.11 (1.23 to 3.62$)$ & - \\
\hline \multicolumn{4}{|c|}{ Travel time to CR centre (min) } \\
\hline \multicolumn{4}{|c|}{ Number of comorbidities } \\
\hline 0 & 1 & 1 & - \\
\hline 1 & 0.37 (0.20 to 0.68$)$ & $0.40(0.19$ to 0.80$)$ & - \\
\hline$\geq 2$ & $0.40(0.15$ to 0.12$)$ & $0.22(0.05$ to 1.01$)$ & - \\
\hline \multicolumn{4}{|l|}{$\mathrm{PCl}$} \\
\hline No & 1 & 1 & - \\
\hline Yes & 3.00 (1.87 to 4.80$)$ & 3.24 (1.85 to 5.66$)$ & - \\
\hline \multicolumn{4}{|l|}{ Impaired renal function† } \\
\hline No & 1 & - & - \\
\hline Yes & $0.21(0.05$ to 0.85$)$ & - & - \\
\hline
\end{tabular}

All independent variables were included in the model as categorical, except age (continuous).

Dash line - this variable was no included in the model.

*Variables were forced into models.

†Impaired renal function was defined as serum creatinine level $\geq 1.5 \mathrm{mg} / \mathrm{dL}$.

$\mathrm{CR}$, cardiac rehabilitation; NER, north-east region; $\mathrm{PCI}$, percutaneous cardiac intervention.

only one private community-based centre. These inequalities are even more prominent because the costs of public centres are partially supported by the NHS, whereas CR programmes running in private centres are not funded by the government. During the study period, however, the private centre in the NER was supported by a specific 
protocol established with the government between 2012 and 2015, covering the costs of CR. Even so, costs were still mentioned as an important barrier to participation which may be explained by the indirect financial costs of participating in CR, namely the high transportation costs especially in more remote areas and for patients of lower socioeconomic status. Furthermore, patients from the Porto district identified delays to initiate the CR programme and lack of awareness of the benefits of CR as the main barriers, whereas limited time availability was identified by patients from both regions. In line with our results, a review that included 34 qualitative studies identified as key the physical barriers, such as lack of transport, financial cost or work commitment, and personal barriers, including embarrassment about participation or lack of perceived benefit of CR. ${ }^{34}$

\section{Limitations}

This study includes a multitude of factors that give important insights about access to CR in Portugal, although the findings should be interpreted in the context of the following potential limitations. First, recall bias may be at play as a result of the amount of time that elapsed between discharge and completion of the 6-month follow-up. However, using information about CR use based on patients' self-report may be more accurate than using physicians' chart. Previous research has shown that despite high concordance between self-reported and chart report, physicians' referral may be influenced by the barriers identified by patients during the CR referral discussion, which may result in lower referral reporting in chart. ${ }^{25}$ Lastly, our results may not be generalised to other settings with different characteristics, although the study was conducted in two hospitals, one university hospital and one tertiary-level hospital, allowing the comparison of different urban and rural settings.

\section{CONCLUSION}

CR remains insufficiently implemented in Portugal, with major inequalities between regions. Our results show clear differences in access among vulnerable subpopulations, such as rural populations, elders, high-risk patients and socioeconomically deprived. The achievement of optimal CR use requires a multilevel approach addressing barriers related to healthcare system, providers and patients in order to improve provision, referral and participation.

Contributors $A A$ conceived and designed the study. MV and $A B$ raised the hypotheses and drafted the first version of the manuscript. MV, $A B, C A$ and $O L$ participated in data collection. MV, AB, AIR, NL and AA analysed and interpreted the data. CA, AR, OL, PD, MJM, IM, NL and AA reviewed the article critically for important intellectual content. All authors read and approved the final manuscript.

Funding This study was supported by FEDER through the Operational Programme Competitiveness and Internationalisation and national funding from the Foundation for Science and Technology-FCT (Portuguese Ministry of Science, Technology and Higher Education) (FCOMP-01-0124-FEDER-028709), under the project 'Inequalities in coronary heart disease management and outcomes in Portugal' (FCT
PTDC/DTP-EPI/0434/2012) and the Unidade de Investigação em EpidemiologiaInstituto de Saúde Pública da Universidade do Porto (EPIUnit) (POCI-01-0145FEDER-006862; ref UID/DTP/04750/2013).

\section{Competing interests None declared.}

Ethics approval The study was approved by the Ethics Committee of Centro Hospitalar de São João and Ethics Committee of Centro Hospitalar de Trás-0sMontes e Alto Douro.

Provenance and peer review Not commissioned; externally peer reviewed.

Data sharing statement Data are available by emailing to the corresponding author at marta.viana@ispup.up.pt.

Open Access This is an Open Access article distributed in accordance with the Creative Commons Attribution Non Commercial (CC BY-NC 4.0) license, which permits others to distribute, remix, adapt, build upon this work non-commercially, and license their derivative works on different terms, provided the original work is properly cited and the use is non-commercial. See: http://creativecommons.org/ licenses/by-nc/4.0/

(c) Article author(s) (or their employer(s) unless otherwise stated in the text of the article) 2018. All rights reserved. No commercial use is permitted unless otherwise expressly granted.

\section{REFERENCES}

1. Amsterdam EA, Wenger NK, Brindis RG, et al. 2014 AHA/ACC guideline for the management of patients with non-ST-elevation acute coronary syndromes: a report of the American college of cardiology/American heart association task force on practice guidelines. Circulation 2014;130:e344-426.

2. O'Gara PT, Kushner FG, Ascheim DD, et al. 2013 ACCF/AHA guideline for the management of ST-elevation myocardial infarction: a report of the American college of cardiology foundation/American heart association task force on practice guidelines. Circulation 2013;127:529-55.

3. Roffi M, Patrono C, Collet JP, et al. 2015 ESC Guidelines for the management of acute coronary syndromes in patients presenting without persistent ST-segment elevation: task force for the management of acute coronary syndromes in patients presenting without persistent st-segment elevation of the European Society of Cardiology (ESC). Eur Heart J 2016;37:267-315.

4. Steg PG, James SK, Atar D, et al. ESC Guidelines for the management of acute myocardial infarction in patients presenting with ST-segment elevation. Eur Heart J 2012;33:2569-619.

5. Anderson L, Thompson DR, Oldridge N, et al. Exercise-based cardiac rehabilitation for coronary heart disease. Cochrane Database Syst Rev 2016:CD001800.

6. Kureshi F, Kennedy KF, Jones PG, et al. Association between cardiac rehabilitation participation and health status outcomes after acute myocardial infarction. JAMA Cardiol 2016;1:980-8.

7. Briffa TG, Eckermann SD, Griffiths AD, et al. Cost-effectiveness of rehabilitation after an acute coronary event: a randomised controlled trial. Med J Aust 2005;183:450-5.

8. Fidan D, Unal B, Critchley J, et al. Economic analysis of treatments reducing coronary heart disease mortality in England and Wales, 2000-2010. QJM 2007;100:277-89.

9. Turk-Adawi K, Sarrafzadegan N, Grace SL. Global availability of cardiac rehabilitation. Nat Rev Cardiol 2014;11:586-96.

10. Abreu A, Bettencourt N, Fontes P. Overview of cardiac rehabilitation in Portugal 2007-2009. Rev Port Cardiol 2010;29:545-58.

11. Silveira C, Abreu A. Cardiac rehabilitation in Portugal: results from the 2013-14 national survey. Rev Port Cardiol 2016;35:659-68.

12. Directorate-General of Health. National Health Plan 2004-2010. 2004 http://1nj5ms2lli5hdggbe3mm7ms5.wpengine.netdna-cdn.com/files/ 2015/08/Volume-1-English-version.pdf.

13. Instituto Nacional de Estatística. Classificação Portuguesa das Profissões. $2011 \mathrm{https}$ ://www.ine.pt/xportal/xmain?xpid=INE\& xpgid=ine_publicacoes\&PUBLICACOESpub_boui=107961853\& PUBLICACOESmodo=2 (accessed 16 Dec 2016).

14. International Labour Office. International Standard Classification of Occupations (ISCO). 2012 http://www.ilo.org/public/english/bureau/ stat/isco/docs/publication08.pdf (accessed 16 Dec 2016).

15. Clark AM, King-Shier KM, Duncan A, et al. Factors influencing referral to cardiac rehabilitation and secondary prevention programs: a systematic review. Eur J Prev Cardiol 2013;20:692-700.

16. Kotseva K, Wood D, De Backer G, et al. Use and effects of cardiac rehabilitation in patients with coronary heart disease: results from the EUROASPIRE III survey. Eur J Prev Cardiol 2013;20:817-26. 
17. Colella TJ, Gravely S, Marzolini S, et al. Sex bias in referral of women to outpatient cardiac rehabilitation? A meta-analysis. Eur J Prev Cardiol 2015;22:423-41.

18. Brown TM, Hernandez AF, Bittner V, et al. Predictors of cardiac rehabilitation referral in coronary artery disease patients: findings from the American heart association's get with the guidelines program. J Am Coll Cardiol 2009;54:515-21.

19. Sumner J, Grace SL, Doherty P. Predictors of cardiac rehabilitation utilization in england: results from the national audit. J Am Heart Assoc 2016;5:e003903.

20. Shanmugasegaram S, Oh P, Reid RD, et al. Cardiac rehabilitation barriers by rurality and socioeconomic status: a cross-sectional study. Int J Equity Health 2013;12:72.

21. Cortés O, Arthur HM. Determinants of referral to cardiac rehabilitation programs in patients with coronary artery disease: a systematic review. Am Heart J 2006;151:249-56.

22. Ko DT, Mamdani M, Alter DA. Lipid-lowering therapy with statins in high-risk elderly patients: the treatment-risk paradox. JAMA 2004;291:1864-70.

23. McAlister FA, Oreopoulos A, Norris CM, et al. Exploring the treatment-risk paradox in coronary disease. Arch Intern Med 2007;167:1019-25.

24. Listerman J, Bittner V, Sanderson BK, et al. Cardiac rehabilitation outcomes: impact of comorbidities and age. J Cardiopulm Rehabil Prev 2011;31:342-8.

25. Grace SL, Gravely-Witte S, Brual J, et al. Contribution of patient and physician factors to cardiac rehabilitation referral: a prospective multilevel study. Nat Clin Pract Cardiovasc Med 2008;5:653-62.

26. Gallagher R, Neubeck L, Du H, et al. Facilitating or getting in the way? The effect of clinicians' knowledge, values and beliefs on referral and participation. Eur J Prev Cardiol 2016;23:1141-50.
27. Gravely-Witte S, Leung YW, Nariani R, et al. Effects of cardiac rehabilitation referral strategies on referral and enrollment rates. Nat Rev Cardiol 2010;7:87-96.

28. Grace SL, Russell KL, Reid RD, et al. Effect of cardiac rehabilitation referral strategies on utilization rates: a prospective, controlled study. Arch Intern Med 2011:171:235-41.

29. Grace SL, Leung YW, Reid R, et al. The role of systematic inpatient cardiac rehabilitation referral in increasing equitable access and utilization. J Cardiopulm Rehabil Prev 2012;32:41-7.

30. Brual J, Gravely-Witte S, Suskin N, et al. Drive time to cardiac rehabilitation: at what point does it affect utilization? Int $J$ Health Geogr 2010;9:27.

31. Bosanac EM, Parkinson RC, Hall DS. Geographic access to hospital care: a 30-minute travel time standard. Med Care 1976;14:616-24.

32. Clark RA, Coffee N, Turner D, et al. Application of geographic modeling techniques to quantify spatial access to health services before and after an acute cardiac event: the cardiac Accessibility and Remoteness Index for Australia (ARIA) project. Circulation 2012;125:2006-14.

33. Buckingham SA, Taylor RS, Jolly K, et al. Home-based versus centre-based cardiac rehabilitation: abridged Cochrane systematic review and meta-analysis. Open Heart 2016;3:e000463.

34. Neubeck L, Freedman SB, Clark AM, et al. Participating in cardiac rehabilitation: a systematic review and meta-synthesis of qualitative data. Eur J Prev Cardiol 2012;19:494-503.

35. De Vos C, Li X, Van Vlaenderen I, et al. Participating or not in a cardiac rehabilitation programme: factors influencing a patient's decision. Eur J Prev Cardiol 2013;20:341-8. 